\title{
Multiple Applications of Ion Beams in Life Science
}

\author{
Atsushi Tanaka \\ Kansai Photon Science Institute, National Institutes for Quantum and Radiological Science and Technology, \\ 8-1-7 Umemidai, Kizugawa-City, Kyoto Pref. 619-0215, Japan; tanaka.atsushi@qst.go.jp
}

Received: 12 September 2019; Accepted: 29 September 2019; Published: 30 September 2019

Welcome to the Special Issue of Quantum Beam Science that features application of ion beams in biology and medicine. The biological application of the ion beams has shown great progress in the past 20 years. Ion beam accelerators have been constructed one after another for the study of physics and fundamental sciences in the 20th century, and medical applications of ion beams advanced circa the 1980s. In the 1990s, several accelerator facilities were established not only for fundamental science but also for medical and biological study. Beyond the basic biological effects of ion beams such as DNA damage, cell death, and mutation induction, the application of ion beams diverges into many branches of life sciences, agriculture, and medical use, such as mutation breeding, developing new varieties in plants and microorganisms; cancer diagnosis by radio isotope (RI)-labeled chemicals; and cancer therapy by use of proton and heavy ion irradiation.

The most important reason for the use of ion beams in biological studies is that their biological effectiveness is the highest among many kinds of radiations or quantum beams. The energy deposition of ion beams into a cell is ten to thousand times higher in comparison with that of gamma rays, $\mathrm{X}$-rays, or electrons. Carbon ion beams, which are mostly used among ion beams, deposit energy efficiently in a target throughout the penetration depth and cause cell death and mutation decisively and locally. As a result, they are well applied to cancer treatment and for developing mutations of new varieties. On the other hand, ion beams colliding with atoms and atomic inner shell electrons happen to generate characteristic $\mathrm{X}$-rays and radioactive nuclides, or radioisotopes (RIs). These characteristic X-rays are used for particle-induced X-ray emission (PIXE) analysis. RIs generated by ion beams tend to become a neutron-deficient nucleus, resulting in electron capture and positron emitting radionuclei, which is useful for the functional imaging of biomolecules.

Included in this Special Issue are some reported examples of the science and technologies essential to ion beam application in life science. The characteristics of mutation induction are analyzed in Arabidopsis as a model plant [1] and in budding yeast as a model of eukaryote cells at the molecular level [2], presenting detailed features of ion beam-induced mutation at the molecular level. Whereas, Satoh and Oono introduced not only the characteristics of mutation induction but also its application in various microorganisms in many industries, including stable food production, sustainable agriculture, development of energy resources, and so on [3]. As cluster ion beams have unique features in energy deposition, it is highly interesting that biological studies using cluster ions were conducted for the first time [4]. Heavy ion microbeam systems have been established because microbeams are necessary not only to elucidate the biological effects of heavy ions by breaking down the non-uniformity of the dose deposition of heavy ions, but also to unravel the function of cells, organs, and tissues of living things contributing to neurobiology, physiology, systems biology, and so on [5].

As an indirect use of ion beams in biological study, PIXE is one of the most successful techniques that could analyze most of the elements in a cell simultaneously at high sensitivity. These PIXE analyses are totally and generally summarized [6] and a unique study of micro-PIXE to indicate $\mathrm{Al}$ and $\mathrm{Si}$ formation in epidermal cells of mature tea leaves is examined [7]. Suzui et al. describe RI imaging systems employed in plant science research. As the investigations in plant sciences require an imaging 
system capable of covering a wide variety of elements, multiple highly versatile technologies are required rather than one state-of-the-art technology for a specific application [8].

The articles announced here include many latest results and techniques. I believe you will enjoy reading about the progress made in the biological application of ion beams in this Special Issue.

Conflicts of Interest: The author declares no conflict of interest.

\section{References}

1. Jo, Y.D.; Kim, J.-B. Frequency and Spectrum of Radiation-Induced Mutations Revealed by Whole-Genome Sequencing Analyses of Plants. Quantum Beam Sci. 2019, 3, 7. [CrossRef]

2. Matuo, Y.; Izumi, Y.; Sakamoto, A.N.; Hase, Y.; Satoh, K.; Shimizu, K. Molecular Analysis of Carbon Ion-Induced Mutations in DNA Repair-Deficient Strains of Saccharomyces cerevisiae. Quantum Beam Sci. 2019, 3, 14. [CrossRef]

3. Satoh, K.; Oono, Y. Studies on Application of Ion Beam Breeding to Industrial Microorganisms at TIARA. Quantum Beam Sci. 2019, 3, 11. [CrossRef]

4. Hase, Y.; Satoh, K.; Chiba, A.; Hirano, Y.; Tomita, S.; Saito, Y.; Narumi, K. Experimental Study on the Biological Effect of Cluster Ion Beams in Bacillus subtilis Spores. Quantum Beam Sci. 2019, 3, 8. [CrossRef]

5. Funayama, T. Heavy-Ion Microbeams for Biological Science: Development of System and Utilization for Biological Experiments in QST-Takasaki. Quantum Beam Sci. 2019, 3, 13. [CrossRef]

6. Ishii, K. PIXE and Its Applications to Elemental Analysis. Quantum Beam Sci. 2019, 3, 12. [CrossRef]

7. Haruyama, Y.; Fujiwara, T.; Yasuda, K.; Saito, M.; Suzuki, K. Localization of Aluminum in Epidermal Cells of Mature Tea Leaves. Quantum Beam Sci. 2019, 3, 9. [CrossRef]

8. Suzui, N.; Tanoi, K.; Furukawa, J.; Kawachi, N. Recent Advances in Radioisotope Imaging Technology for Plant Science Research in Japan. Quantum Beam Sci. 2019, 3, 18. [CrossRef] 